\title{
Modulation of Protein Kinase C Isozymes and Substrates by Lithium: The Role of Myo-inositol
}

\author{
Husseini K. Manji, M.D., F.R.C.P.C., Yuly Bersudsky, Ph.D., Guang Chen, M.D.,
} Robert H. Belmaker, M.D., and William Z. Potter, M.D., Ph.D.

\begin{abstract}
Lithium is the most effective treatment for reducing both the frequency and severity of recurrent affective episodes, but despite extensive research, the molecular mechanisms underlying its therapeutic actions have not been fully elucidated. Signal transduction pathways are in a pivotal postion in the central nervous system, able to affect the functional balance between neurotransmitter systems and have clearly been demonstrated to be targets of lithium's actions. We investigate the hypothesis that the action of chronic lithium on PKC isozymes and substrates may be secondary to its potent effect in inhibiting the recycling of inositol. Rats received lithium for 3 weeks and also myoinositol or saline twice daily via intracerebroventricular (ICV) injections. There was a significant interaction
\end{abstract}

between chronic lithium and myo-inositol administration, with the chronic ICV administration of myo-inositol attenuating lithium's effects on PKC $\alpha, P K C \epsilon$, and on pertussis toxin-catalyzed [ ${ }^{32} P I A D P$-ribosylation. These results suggest that the effects of chronic lithium on signal transduction pathways may stem initially from its inhibition of inositol-1-phosphatase. Given the critical role of $P K C$ isozymes and $G$ proteins in modulating intracellular cross-talk between neurotransmitter systems and thereby the integrative functions of the CNS, future studies using other inhibitors of inositol monophosphatases are warranted, and offer the hope for the development of more potent and more rapidly acting mood-stabilizing drugs. [Neuropsychopharmacology 15:370-381, 1996]
KEY WORDS: Lithium; Manic-depressive; Protein kinase C; G proteins; Myo-inositol

Lithium, a simple monovalent cation, has demonstrated efficacy in the treatment of manic-depressive illness and is the most effective treatment for reducing both the fre-

From the Molecular Pathophysiology Program, Schizophrenia and Mood Disorders Clinical Research Division, Departments of Psychiatry and Pharmacology (HKM, GC), Wayne State University School of Medicine, Detroit, MI; the Beersheva Mental Health Center (YB, RHB), Beersheva, Israel, and the Section on Clinical Pharmacology (WZP), National Institute of Mental Health, Bethesda, MD.

Address correspondence to: Husseini K. Manji, M.D., F.R.C.P.C., Director, Schizophrenia and Mood Disorders Clinical Research Division, Department of Psychiatry and Behavioral Neurosciences, Wayne State University School of Medicine, UHC 9B, 4201 St. Antoine Boulevard, Detroit, MI 48201.

Received July 14, 1995; revised November 3, 1995; accepted November 9, 1995. quency and severity of recurrent affective episodes (reviewed in Goodwin and Jamison 1990). Despite lithium's remarkable efficacy and decades of use, the molecular mechanisms underlying its therapeutic actions have not fully been elucidated. In recent years, it has become increasingly clear that rather than any single neurotransmitter system being responsible for depression or mania, multiple interacting and overlapping systems are involved in regulating mood, and that most effective drugs such as lithium do not work on any particular neurotransmitter system in isolation but, rather, affect the functional balance between interacting systems (Janowsky et al. 1972; Avissar et al. 1988; Manji et al. 1995). Signal transduction pathways are in a pivotal position in the central nervous system (CNS), can affect the functional balance between neurotransmitter systems, and have therefore been postulated to represent the targets underlying lithium's efficacy in treating both poles of 
manic-depressive illness (Manji 1992; Jope and Williams 1994).

There is now considerable evidence that the chronic administration of "therapeutic" concentrations of lithium affects the function of second messenger-generating systems in the brain (Ebstein et al. 1976; reviewed in Mork et al. 1992; Jope and Williams 1994; Manji et al. 1995). At therapeutically relevant concentrations, lithium, is a potent inhibitor of inositol monophosphatase $\left(\mathrm{K}_{\mathrm{i}}, 0.8 \mathrm{mM}\right)$ and results in an accumulation of inositol1 -monophosphate $(\operatorname{Ins}(1) \mathrm{P})$ as well as a reduction in free inositol (Allison and Stewart 1971; Hallcher and Sherman 1980). Because the ability of a cell to maintain sufficient supplies of myo-inositol is crucial to the resynthesis of the phosphoinositides and the maintenance and efficiency of signaling, and because the mode of enzyme inhibition is uncompetitive, lithium's effects have been postulated to be most pronounced in systems undergoing the highest rate of $\mathrm{PIP}_{2}$ hydrolysis (Berridge et al. 1982, 1989). Because the brain has limited access to inositol other than that derived from recycling of inositol phosphates, it has been suggested that a physiological consequence of lithium's action is derived through a depletion of free inositol (Berridge et al., 1982, 1989). Chronic lithium administration has been demonstrated to reduce agonist-stimulated phosphoinositide hydrolysis in rat brain in some studies (Casebolt and Jope 1989; Kendall and Nahorski 1987; Godfrey et al. 1989) and more recently to have multiple effects on the PI second messenger-generating system, including at the level of protein kinase C (PKC; reviewed in Jope and Williams 1994 and Manji and Lenox 1994). In recent studies, chronic lithium administration has been demonstrated to significantly reduce the levels of membrane-associated PKC $\alpha$ and PKC $\epsilon$ (Manji et al. 1993) (Masana MI, Chen G, Shorts L, Potter WZ, Manji HK (1995): Attenuation of PKC induced cyclic AMP increases in rat brain by chronic lithium: An in vivo microdialysis study. Under revision.) and of a major PKC phosphoprotein substrate, MARCKS (myristoylated alanine-rich C kinase substrate) in the rat hippocampus (Lenox et al. 1992).

The mechanism(s) by which lithium produces the isozyme-selective decreases in the immunolabeling of PKC $\alpha$ and $\epsilon$ and in the levels of MARCKS is presently unclear. It is noteworthy, however, that as a result of inositol depletion in the presence of lithium, metabolites within the phospholipid portion of the hydrolytic pathway that include diacylglycerol (DAG), phosphatidic acid (PA), and cytidine monophosphate-phosphatidate (CMP-PA) have been shown to be significantly elevated. This effect can be prevented by the presence of high concentrations of inositol (Drummond and Raeburn 1984; Downes and Stone 1986; Brami et al. 1991a, b). This might be expected as the end metabolite in this pathway, (i.e., CMP-PA) requires free myo-inositol to resynthesize inositol phosphates for the regenera- tion of $\mathrm{PIP}_{2}$ (Rana and Hokin 1990; Fisher et al. 1992). Because PKC activation is often followed by its rapid proteolytic degradation (Young et al. 1987), a prolonged increase in DAG levels by chronic lithium may lead to an increased membrane translocation and subsequent degradation and downregulation of PKC isozymes. Moreover, recent studies in fibroblasts have demonstrated that activating PKC results in a significant reduction in the levels of both MARCKS protein and mRNA (Erusalimsky et al. 1991; Linder et al. 1992). Thus, the reduction in hippocampal MARCKS observed following chronic lithium administration may similarly be mediated via a lithium-induced increase in DAG levels and subsequent activation of PKC isozymes.

In addition to its effects on PKC outlined, there is also considerable evidence that chronic lithium administration affects $\mathrm{G}$ protein function in both rodents and human subjects (Mork et al. 1992; Jope and Williams 1994; Manji et al. 1995). In a recent series of studies, the attenuation of the function of the inhibitory $G$ protein $\left(G_{i}\right)$ was accompanied by a significant increase in pertussis toxin-catalyzed [ $\left.{ }^{32} \mathrm{P}\right] \mathrm{ADP}$-ribosylation in rat brain (Masana et al. 1995). As with most other studies (reviewed in Manji et al. 1995), these investigators did not observe alterations in the amounts of $\alpha_{\mathrm{s}}, \alpha_{\mathrm{i} 1-3}$, or $\alpha_{\mathrm{o}}$. Because pertussis toxin selectively ADP-ribosylates the undissociated, inactive $\alpha \beta \gamma$ heterotrimeric form of $G_{i}$ and $G_{0}(\mathrm{Ui} 1990)$, it has been postulated that lithium inactivates $G_{i}$ by stabilizing the inactive conformation (Hsiao et al. 1992; Masana et al. 1995, under revision). At present, the molecular mechanism(s) underlying lithium's effects on $G$ proteins remains to be fully established. There is some evidence for acute in vitro effects of lithium on G proteins (Avissar et al. 1988; Greenwood and Jope 1994), but many of lithium's chronic ex vivo effects persist after washing of the membranes and are therefore likely attributable to an indirect posttranslational modification of the G proteins (Jope and Williams 1994; Manji et al. 1995). Because G protein function is known to be regulated by phosphorylation (Houslay 1991), and because considerable evidence has shown that lithium affects PKC, one potential mechanistic explanation for lithium's effects on $G$ protein subunit dissociation is phosphorylation by PKC.

In the present study, we sought to investigate the hypothesis that the action of chronic lithium on PKC isozymes and substrates may be secondary to its potent effect in inhibiting the recycling of inositol. Previous studies have shown that the administration of pharmacological concentrations of extracellular inositol attenuates some of lithium's biochemical, behavioral, and toxic effects (Busa and Gimlich 1989; Godfrey et al. 1989; Pontzer and Crews 1990; Kofman and Belmaker 1993; Tricklebank et al. 1991; Kofman et al. 1991; 1993). We have therefore investigated the role of myo-inositol supplementation on lithium-induced alterations in PKC 
isozymes and PKC substrates and on pertussis toxincatalyzed [ $\left.{ }^{32} \mathrm{P}\right] \mathrm{ADP}-$ ribosylation. We have found that the daily intracerebroventricular administration of myoinositol attenuates several of chronic lithium's effects on signal transduction pathways.

\section{MATERIALS AND METHODS}

\section{Animals}

Male Sprague-Dawley rats (weight 250 to $350 \mathrm{~g}$ ) were housed four per cage and exposed to a 12-hour light/ dark cycle. The control group $(n=16)$ was fed ordinary rat chow, and the treatment group $(n=16)$ was fed $\mathrm{Li}_{2} \mathrm{CO}_{3}$-supplemented chow $(0.2 \%$ by weight $)$ for 3 weeks. Serum lithium concentration were determined by flame photometry in carotid blood obtained at sacrifice by decapitation.

For the purposes of myo-inositol administration, all the rats $(n=32)$ were anesthetized with Nembutal (50 $\mathrm{mg} / \mathrm{Kg}$ ) and implanted with guide cannulae into the dorsal part of the lateral ventricle using standard stereotaxic procedures. Coordinates for the cannula were $0.8 \mathrm{~mm}$ posterior to the bregma, $1.4 \mathrm{~mm}$ lateral to midline, and $5 \mathrm{~mm}$ below skull surface. At least 7 days of recovery was allowed following surgery. Rats were allowed to recover and were observed to behave normally with the chronic indwelling intracerebroventricular (ICV) cannula prior to initiation of lithium and/or myo-inositol treatment. Myo-inositol was dissolved in warm artificial cerebrospinal fluid (CSF) $(147 \mathrm{mM} \mathrm{NaCl}, 2.5 \mathrm{mM}$ $\mathrm{KCl}, 1.6 \mathrm{mM} \mathrm{MgCl} 2,1.7 \mathrm{mM} \mathrm{CaCl}_{2}$, and $2.2 \mathrm{mM}$ glucose). Rats were injected twice daily for 3 weeks with either $10 \mathrm{mg}$ myo-inositol [ $250 \mu \mathrm{g} / \mu \mathrm{l}$ in a volume of $40 \mu \mathrm{l}$; $(n=16 ; 8$ lithium treated, 8 control) $]$ or artificial CSF ( $n=$ 16; 8 lithium treated, 8 control) over a period of 3 minutes using a Koehlin 100- $\mu \mathrm{l}$ microsyringe, via an injection cannula that protruded $0.5 \mathrm{~mm}$ below the guide cannula. This treatment paradigm resulted in the generation of four distinct groups: lithium-artificial cerebrospinal fluid (CSF; $n=8)$, lithium-inositol $(n=8)$, control-CSF $(n=8)$, and control-inositol $(n=8)$. The time course of twice daily ICV inositol administration and dose of $10 \mathrm{mg}$ was based on studies of Bersudsky et al. (1993), where Li-pilocarpine seizures required $10 \mathrm{mg}$ for full reversal of effects and the effect of inositol was present after 12 hours, but gone by 24 hours.

\section{Immunoblotting}

For the immunoblotting studies, the rat brains were removed immediately upon decapitation, dissected on ice, and hippocampi and frontal cortices were rapidly frozen in dry ice and stored at $-80^{\circ} \mathrm{C}$. For the subse- quent studies, each animal was treated as an individual sample, and there was no pooling of tissues. For Western blot anlaysis, the tissue was homogenized in $20 \mathrm{mM}$ Tris buffer ( $\mathrm{pH} 7.4$ ) containing $2 \mathrm{mM}$ EDTA, $0.5 \mathrm{mM}$ EGTA, $10 \mathrm{mg} / \mathrm{ml}$ leupeptin, $10 \mathrm{mg} / \mathrm{ml}$ apoprotinin, 0.2 $\mathrm{mM}$ phenylmethylsulfonylfluoride, using a glass-glass tissue grinder (15 strokes). The homogenate was centrifuged at $1,000 \mathrm{~g}$ for 5 minutes in a Beckman table centrifuge. The resultant supernatant was centrifuged at $45,000 \mathrm{~g}$ for 1 hour at $4^{\circ} \mathrm{C}$ in a Beckman TL-100 centrifuge. The pellet (particulate fraction) was resuspended in the original volume of homogenization buffer. The supernatant (soluble fraction) was centrifuged again at $45,000 \mathrm{~g}$ for 2 hours to remove residual particulate fraction. An aliquot was taken for protein determination, and the fractions were then frozen at $-80^{\circ} \mathrm{C}$ in plastic tubes. Protein concentration was determined by BioRad Protein Assay kit (Bio-Rad, Richmond, CA) using bovine serum albumin as standard. PKC isozyme and PKC substrate (MARCKS and GAP-43) immunoblotting was performed on both cytosolic and particulate fractions. The linearity of the particulate and soluble fraction protein concentration for Western blotting was previously ascertained by resolution of selected concentrations of protein (between 10 and $200 \mu \mathrm{g}$ ). Subsequent PKC isozyme, MARCKS, and GAP-43 immunoblotting was performed using particulate or soluble protein concentrations demonstrated to be within the linear range for Western blotting using previously described methods (Manji et al. 1993; Masana et al. 1995). In brief, $50 \mu \mathrm{g}$ of particulate and soluble fraction samples were subjected to SDS-PAGE on $10 \%$ polyacrylamide gels $(5 \%$ stacking gel) according to the method of Laemmli (1970). Proteins resolved on SDS-PAGE were then electrophoretically transferred to nitrocellulose membranes. Nonspecific binding on the nitrocellulose was blocked with lowz-detergent "blotto" containing $50 \mathrm{mM}$ TRIS ( $\mathrm{pH}$ 8.0), $2 \mathrm{mM} \mathrm{CaCl}, 80 \mathrm{mM} \mathrm{NaCl}, 5 \%$ nonfat dry milk, $0.2 \% \mathrm{NP}-40$, and $0.02 \% \mathrm{NaN}_{3}$. Blots were then incubated at room temperature overnight with monoclonal anti-PKC isozyme antibodies [anti-PKC $\alpha$, anti-PKC $\beta(\mathrm{I} / \mathrm{II})$, anti-PKC $\gamma$, Seikagaku America, St. Petersburg, FL; anti-PKC $\delta$, anti-PKC $\epsilon$, anti-PKC $\zeta$, GIBCO, BRL Bethesda, MD], anti-MARCKS antibody (kindly donated by Dr. Jitendra Patel), and anti-GAP 43 antibody (Boeringer, Mannheim). The antibodies for all PKC isoforms, MARCKS, and GAP-43 were all diluted $1: 1,000$ in low-detergent blotto prior to use. Blots were washed three times, and in the case of the anti-PKC $\alpha$, anti-PKC $\beta(\mathrm{I} / \mathrm{II})$, anti-PKC $\gamma$ antibodies, incubated with a second antibody, antimouse IgG from rabbit $(1 \mathrm{mg} / \mathrm{ml} ; 1: 200$ dilution; Cappel, Malvern, PA) for 2 hours. After three washes, the nitrocellulose sheets were incubated with $1 \times 10^{6} \mathrm{cpm}\left[{ }^{125} \mathrm{I}\right]$ protein A (Amersham) for 1 hour. The labeled blots were then washed, dried, and exposed to 
X-ray film with intensifying screens at $-80^{\circ} \mathrm{C}$. Quantitation of the immunoblots was performed by densitometric scanning of the autoradiograms using an Image Analysis system (NIH 1.47). An aliquot of pooled "standard" rat brain soluble or particulate fraction was run on one lane of each gel, and the immunolabeling was calculated relative to this standard. Data were normalized against the standard rat brain fractions, which were run on all blots to minimize between-blot variability.

\section{Pertussis Toxin-Catalyzed [ ${ }^{32}$ P]ADP-Ribosylation}

Pertussis toxin (PT)-catalyzed labeling of rat cortical or hippocampal membrane $G_{i} / G_{o}$ was performed using previously described methods (Masana et al. 1995); in brief, rat brain membranes were prepared as described and were incubated at $37^{\circ} \mathrm{C}$ for 60 minutes in a final volume of $0.2 \mathrm{ml}$, containing $100 \mathrm{mM}$ potassium phosphate buffer ( $\mathrm{pH} 7.5), 100 \mathrm{mM}$ thymidine, $10 \mathrm{mM}$ arginine, 0.5 $\mathrm{mM}$ ATP, $50 \mu \mathrm{M}$ GTP, and $10 \mu \mathrm{M}\left[\alpha-{ }^{32} \mathrm{P}\right]-\mathrm{NAD}(25 \mathrm{Ci} /$ mMol, New England Nuclear, Boston) with activated toxin. Before incubation, pertussis toxin $(5 \mu \mathrm{g} /$ tube, obtained from List Biological Laboratories, Campbell, CA) was activated with $10 \mathrm{mM}$ dithiothreitol for 30 minutes at $37^{\circ} \mathrm{C}$. The linearity of the plasma membrane protein concentration for quantitation of PT-catalyzed [ $\left.{ }^{32} \mathrm{P}\right]$ labeling was previously ascertained to be between 20 and $400 \mu \mathrm{g}$. Thus, the present studies were performed using protein concentrations of 50 to $100 \mu \mathrm{g}$, with PT-catalyzed [ $\left.{ }^{32} \mathrm{P}\right] \mathrm{ADP}$-ribosylation of all samples performed in duplicate. Labeled membranes were washed in icecold buffer, and proteins were solubilized and subjected to SDS-PAGE on $10 \%$ polyacrylamide gels using the method of Laemmli (1970). The gels were dried and exposed to an X-ray film (Kodak X-omat, Rochester, NY) with an intensifying screen at $-80^{\circ} \mathrm{C}$. Molecular weights of labeled protein bands were estimated by comparison with molecular weight standards. An aliquot of pooled "standard" rat brain membranes was run on one lane of every gel, and the quantitation of $\left[{ }^{32} \mathrm{P}\right]$ incorporation was calculated relative to this standard.

\section{Materials}

PDBu were obtained from Sigma Chemical Company (St. Louis, MO). PKC $\alpha, \beta$, and $\gamma$ antibodies were from Seikagaku Kogyo Co., Ltd (Rockville, MD). PKC $\delta, \zeta$, and $\epsilon$ antibodies were from GIBCO, BRL (Bethesda, MD). Rabbit anti-mouse IgG was from Organon Teknika Co. (Durham, NC). ${ }^{125} \mathrm{I}$-Protein A was from Amersham (Arlington Heights, IL). The protein assay kit was from BioRad Laboratories (Richmond, CA). All other chemicals were of analytical grade.

\section{RESULTS}

Chronic ICV administration of myo-inositol was without effect on plasma lithium levels (ICV CSF group, $0.712 \pm 0.08 ; \mathrm{ICV}$ inositol group, $0.713 \pm 0.07 \mathrm{mM}$; $\mathrm{p}$ NS). Visual examination of the rat brains did not reveal any gross damage of the frontal cortices or hippocampi or enlargement of ventricles from chronic cannula implantation or myo-inositol administration.

\section{Lithium Regulation of PKC Isozymes in the Hippocampus}

These experiments were designed to assess the effects of chronic lithium and/or myo-inositol administration on the levels of both membrane-associated (Table 1) and cytosolic PKC isozymes in the hippocampus. A twoway analysis of variance (ANOVA) was conducted for each of the PKC isozymes $(\alpha, \beta(\mathrm{I} / \mathrm{II}), \gamma, \delta, \epsilon$, and $\zeta)$. A significant interaction between lithium and inositol was observed for membrane-associated PKC $\alpha\left(\mathrm{F}_{1,28}=6.28\right.$; $p<.02)$. Post hoc analysis revealed a significant difference between the levels of membrane-associated PKC $\alpha$ in the myo-inositol-treated group compared to either the control animals $(t=2.27 ; \mathrm{df}=14 ; p<.05)$ or the animals coadministered lithium and myo-inositol $(t=$ $2.36 ; \mathrm{df}=14 ; p<.05)$. The lithium-treated group exhibited modestly lower levels of membrane-associated

Table 1. Hippocampal Membrane PKC Isozymes

\begin{tabular}{llccr}
\hline Measure & \multicolumn{1}{c}{ Control } & Lithium & Lithium/Inositol & \multicolumn{1}{c}{ Inositol } \\
\hline PKC $\alpha$ & $94.7(29.15)$ & $79.7(23.6)$ & $95.37(23.69)$ & $63.91(29.70)$ \\
PKC $\beta$ & $89.25(17.97)$ & $83.99(19.4)$ & $87.64(14.28)$ & $78.63(15.12)$ \\
PKC $\gamma$ & $107.0(32.34)$ & $76.48(22.05)$ & $110.88(44.77)$ & $77.17(29.31)$ \\
PKC $\delta$ & $119.41(18.82)$ & $125.97(17.93)$ & $128.95(11.98)$ & $119.34(16.78)$ \\
PKC $\epsilon$ & $106.2(28.5)$ & $76.5(22.1)$ & $118.1(26.9)$ & $76.49(19.26)$ \\
PKC $\zeta$ & $116.65(21.87)$ & $97.50(24.57)$ & $112.13(22.3)$ & $101.71(32.74)$ \\
\hline
\end{tabular}

Rats were treated with lithium and/or myo-inositol for 3 weeks, and hippocampal cytosolic and membrane fractions were subjected to Western blotting with selective anti-PKC $\alpha$, anti-PKC $\beta(I, I I)$, anti-PKC $\gamma$, anti-PKC $\delta$, anti-PKC $\epsilon$, and anti-PKC $\zeta$ isozyme antibodies. Quantitation of the immunoblots was performed by densitometric scanning of the autoradiograms and was normalized to a pooled standard. 
PKC $\alpha$ than control animals, which did not reach statistical significance in the present study (Con, $94.7 \pm$ 29.15; Lith, $79.7 \pm 23.6$ OD units; $p=.2$ ). The largest interaction between lithium and inositol was observed for membrane-associated PKC $\epsilon\left(\mathrm{F}_{1,27}=9.53 ; p=.005\right)$. The lithium-treated animals demonstrated lower levels of membrane-associated PKC $€$ (Lith, $76.5 \pm 22.1$ OD units) than either the control animals (Con, $106.2 \pm 28.5$; $p=.07)$ or the animals coadministered lithium and myo-inositol (Lith + Inos, $118.1 \pm 26.93 ; p=.01)$. The levels of membrane-associated PKC $\epsilon$ in the myo-inositol-treated group was also significantly different from that in the animals coadministered lithium and myoinositol $(t=2.84 ; \mathrm{df}=14 ; p=.02)$. There were no significant interactions between lithium and myo-inositol observed for hippocampal membrane-associated PKC $\beta(\mathrm{I} / \mathrm{II}), \boldsymbol{\delta}$, or $\zeta$. Similarly, there were no significant interactions between lithium and myo-inositol observed for the levels of any of the PKC isozymes $(\alpha, \beta$ (I/II), $\gamma, \boldsymbol{\delta}, \epsilon$, and $\zeta$ ) in the cytosolic fraction (data not shown).

\section{Lithium Regulation of PKC Isozymes in the Frontal Cortex}

These experiments were designed to assess the effects of chronic lithium and/or myo-inositol administration on the levels of both membrane-associated (Table 2) and cytosolic PKC isozymes in the frontal cortex. A two-way ANOVA was conducted for each of the PKC isozymes $(\alpha, \beta(\mathrm{I} / \mathrm{II}), \gamma, \delta, \epsilon$, and $\zeta)$. In contrast to the results seen in the hippocampus, a significant interaction between lithium and inositol was observed only for membraneassociated PKC $\left(\beta(\mathrm{I} / \mathrm{II})\right.$ in the frontal cortex $\left(F_{1,28}=\right.$ $4.61 ; p<.05)$; post hoc analyses revealed that this was due to the significantly higher levels of membrane-associated PKC $\beta(\mathrm{I} / \mathrm{II})$ in the animals coadministered lithium and myo-inositol treated compared to any of the other groups. In this brain region, although the lithiumtreated group exhibited lower levels of membrane-associated PKC $\propto$ (Con; $99.67 \pm 40.3$; Lith, $83.7 \pm 27.7$ OD units) and PKC $\epsilon$ (Con, 95.8 \pm 31.3; Lith, $79.1 \pm 20.3$ OD units) than for control animals, these decreases did not reach statistical significance. Similar to what was observed in the hippocampus, there were no significant interactions between lithium and myo-inositol observed for the levels of any of the cystosolic PKC isozymes $(\alpha$, $\beta,(\mathrm{I} / \mathrm{II}), \gamma, \delta, \epsilon, \zeta)$ in the frontal cortex (data not shown).

\section{Lithium Regulation of PKC Substrates in Hippocampus}

These experiments sought to investigate the effects of chronic lithium and/or myo-inositol administration on the levels of the major PKC substrates MARCKS and GAP-43 in the hippocampus (Figure 1A shows a representative immunoblot). A two-way ANOVA revealed a significant interaction between lithium and inositol with respect to MARCKS immunolabeling in the particulate fraction $\left(F_{1,28}=4.89 ; p<.04\right)$. Post hoc analyses revealed a significant difference between the levels of MARCKS in the myo-inositol-treated group compared to the animals coadministered lithium and myo-inositol $(t=2.087 ; \mathrm{df}=14 ; p<.05)$. The lithium-treated group exhibited lower levels of MARCKS than did control animals, which did not reach statistical significance (Con, $104.45 \pm 15.54$; Lith, $81.57 \pm 15.55$ OD units; $p=.3$ ). There were no significant interactions between lithium and inositol treatment on the levels of GAP- 43 in the particulate fraction (Figure 1B). There were no significant interactions between lithium and inositol treatment on the levels of either MARCKS or GAP-43 in the hippocampal soluble fraction (not shown).

\section{Lithium Regulation of PKC Substrates in Frontal Cortex}

We investigated the effects of chronic lithium and/or myo-inositol administration on the levels of the major PKC substrates MARCKS and GAP-43 in the frontal cortex (see Figure 2A for a representative immunoblot). There were no significant interactions between lithium and inositol treatment on the levels of MARCKS in the particulate fraction $\left(F_{1,28,}=0.242 ; p=.6\right)$. A near-significant interaction between lithium and inositol was ob-

Table 2. Cortical Membrane PKC Isozymes

\begin{tabular}{lcccr}
\hline Measure & Control & Lithium & Lithium/Inositol & \multicolumn{1}{c}{ Inositol } \\
\hline PKC $\alpha$ & $99.67(40.3)$ & $83.7(27.7)$ & $95.37(23.69)$ & $83.91(29.70)$ \\
PKC $\beta$ & $82.83(18.3)$ & $83.23(9.64)$ & $112.69(14.28)$ & $74.56(15.37)$ \\
PKC $\gamma$ & $113.16(38.76)$ & $118.88(16.9)$ & $116.58(44.45)$ & $101.08(29.31)$ \\
PKC $\delta$ & $90.1(24.65)$ & $92.03(38.04)$ & $103.15(38.88)$ & $88.67(18.15)$ \\
PKC $\epsilon$ & $95.8(31.3)$ & $79.01(20.3)$ & $118.1(26.9)$ & $76.49(19.26)$ \\
PKC $\zeta$ & $106.20(39.64)$ & $111.79(27.72)$ & $110.79(22.3)$ & $100.58(21.69)$ \\
\hline
\end{tabular}

Rats were treated with lithium and/or myo-inositol for 3 weeks, and frontal cortical cytosolic and membrane fractions were subjected to Western blotting with selective anti-PKC $\alpha$, anti-PKC $\beta(\mathrm{I}, \mathrm{II})$, anti-PKC $\gamma$, anti-PKC $\delta$, anti-PKC $\epsilon$, and anti-PKC $\zeta$ isozyme antibodies. Quantitation of the immunoblot was performed by densitometric scanning of the autoradiograms and was normalized to a pooled standard. 
Figure 1. Effects of chronic lithium and/or myo-inositol on immunolabeling of PKC substrates in the hippocampus. Rats were treated with lithium \pm myo-inositol for 3 weeks, and cytosolic and membrane fractions from hippocampi were prepared and subjected to SDSPAGE on $10 \%$ polyacrylamide gels. Proteins thus resolved were electrophoretically transferred to nitrocellulose membranes; blots were incubated overnight with anti-MARCKS and anti-GAP-43 antibodies, followed by [ $\left.{ }^{125} \mathrm{I}\right]$ protein A. Dried blots were exposed to X-ray film with intensifying screens at $-80^{\circ} \mathrm{C}$. Quantitation of the immunoblots was performed by densitometric scanning of the autoradiograms. An aliquot of pooled "standard" rat brain cytosolic or particulate fraction was run on one lane of each gel, and the immunolabeling was normalized to this standard. (A) Representative immunoblot of MARCKS and GAP-43. (B) Effects of chronic lithium \pm myo-inositol administration on immunolabeling of MARCKS and GAP-43 ( $n=8$ in each group). *, Significant interaction between lithium and inositol with respect to MARCKS immunolabeling in the particulate fraction (two-way ANOVA: $F_{1,28}=$ 4.89; $p<.04)$, li, lithium treated; Con, control; inos, myo-inositol treated; $M A R C K S$, myristoylated alanine rich $C$ kinase substrate; $G A P-43$, growth-associated protein.
GAP-43

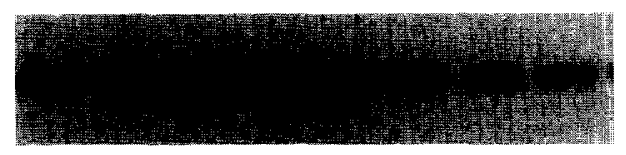

MARCKS

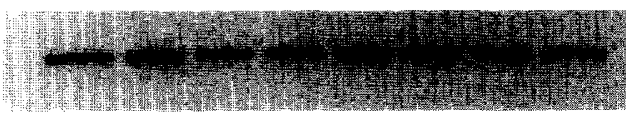

A

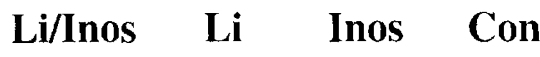

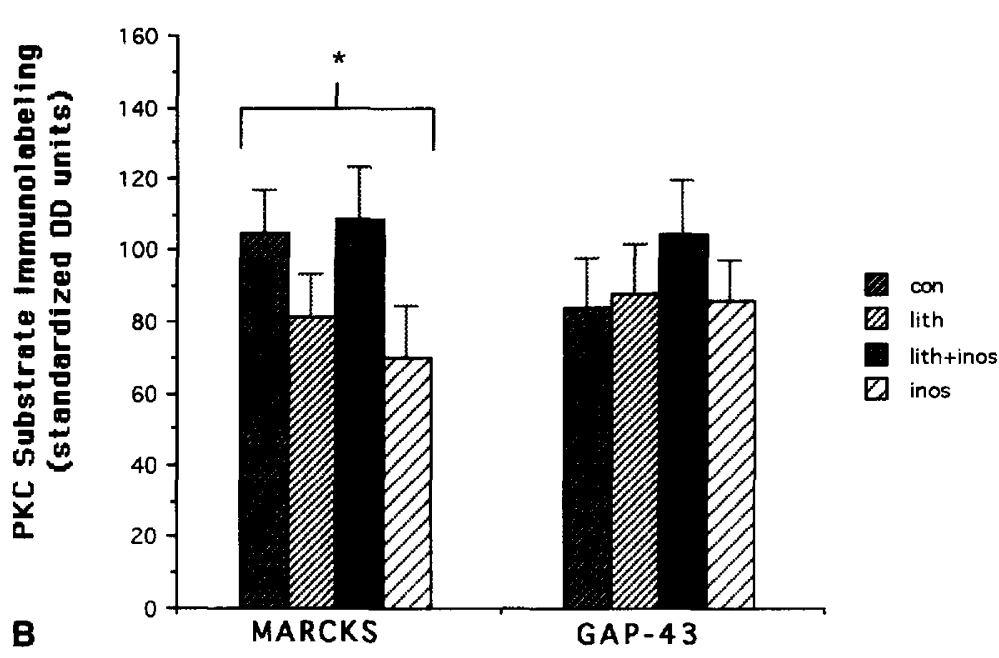

served for GAP-43 in the particulate fraction $\left(F_{1,28}=\right.$ 3.86; $p=.06$ ). Post hoc analyses revealed a significant difference between the levels of GAP-43 in the group of animals coadministered lithium and myo-inositol compared to the myo-inositol-treated group $(t=2.188 ; \mathrm{df}=$ $14 ; p<.05)$ and a trend toward statistical significance compared to the lithium-treated group $(t=1.702 ; \mathrm{df}=$ $14 ; p=.09$; Figure 2B). There were no significant interactions between lithium and inositol treatment on the levels of either MARCKS or GAP-43 in the soluble fraction (not shown).

\section{Effects of Chronic Lithium on Pertussis-Catalyzed $\left[{ }^{32} \mathrm{P}\right]$ ADP-Ribosylation}

These experiments looked into the effects of chronic lithium and/or myo-inositol administration on pertussis-catalyzed [ $\left.{ }^{32} \mathrm{P}\right] \mathrm{ADP}$-ribosylation (an indirect measure of the conformational state of $G_{i} / G_{o}$ ).

Hippocampus. A representative autoradiogram of PTcatalyzed [ $\left.{ }^{32} \mathrm{P}\right] \mathrm{ADP}$-ribosylation (ADPR) in hippocampal membranes is shown in Figure 3A. A two-way ANOVA revealed a highly significant interaction between chronic lithium and inositol treatment with respect to PT-catalyzed [ $\left.{ }^{32} \mathrm{P}\right] \mathrm{ADPR}$ in the hippocampus $\left(F_{1,28}=9.40 ; p<.004\right)$. The lithium-treated group exhibited a significantly higher PT-catalyzed $\left[{ }^{32} \mathrm{P}\right] \mathrm{ADPR}$ than for either the control group (Con; 2,140 \pm 1015; Lith, $3,694 \pm 1,236$ OD units; $t=2.76, \mathrm{df}=14, p=.01$ ), or the myo-inositol treated group (INOS: $2,083 \pm 1,037$ OD units; $t=2.86, \mathrm{df}=14, p<.01$ ). PT-catalyzed [ $\left.{ }^{32} \mathrm{P}\right] \mathrm{ADPR}$ in the group coadministered myo-inositol and lithium was not significantly different from the control group, suggesting that myo-inositol attenuates lithium's effects. Myo-inositol administration alone was almost completely without effect on PT-catalyzed [ $\left.{ }^{32} \mathrm{P}\right] \mathrm{ADPR}$ in the hippocampus (Con; 2,140 $\pm 1,015$; Inos, $2,083 \pm 1,037$ OD units; $t=0.1, \mathrm{df}=14, p=.92$; Figure $3 \mathrm{~B}$ ).

Frontal Cortex. A representative of autoradiogram of PT-catalyzed [ $\left.{ }^{32} \mathrm{P}\right] \mathrm{ADP}$-ribosylation in frontal cortical membranes is shown in Figure 4A. A two-way ANOVA also revealed a significant interaction between chronic lithium and inositol treatment with respect to PT-catalyzed [ $\left.{ }^{32} \mathrm{P}\right] \mathrm{ADPR}$ in the frontal cortex $\left(F_{1,28}=6.74 ; p<\right.$ $.05)$. The lithium-treated group exhibited a significantly higher PT-catalyzed [ ${ }^{32}$ P]ADPR labeling than the myoinositol-treated group (Lith, 2,865 \pm 913; Inos, 1,815 \pm 717 OD units; $t=2.09$, df $=14, p<.05$ ) and near-significant increases compared to the control group (Con, $1,982 \pm 899$ OD units; $t=1.75, \mathrm{df}=14, p=.09$ ). Myoinositol administration alone was almost completely without effect on PT-catalyzed [ ${ }^{32}$ P]ADPR (Con, 1,982 \pm 899 ; Inos, $1,815 \pm 717$ OD units; $t=0.33, \mathrm{df}=14, p=.74$; Figure 4B). 


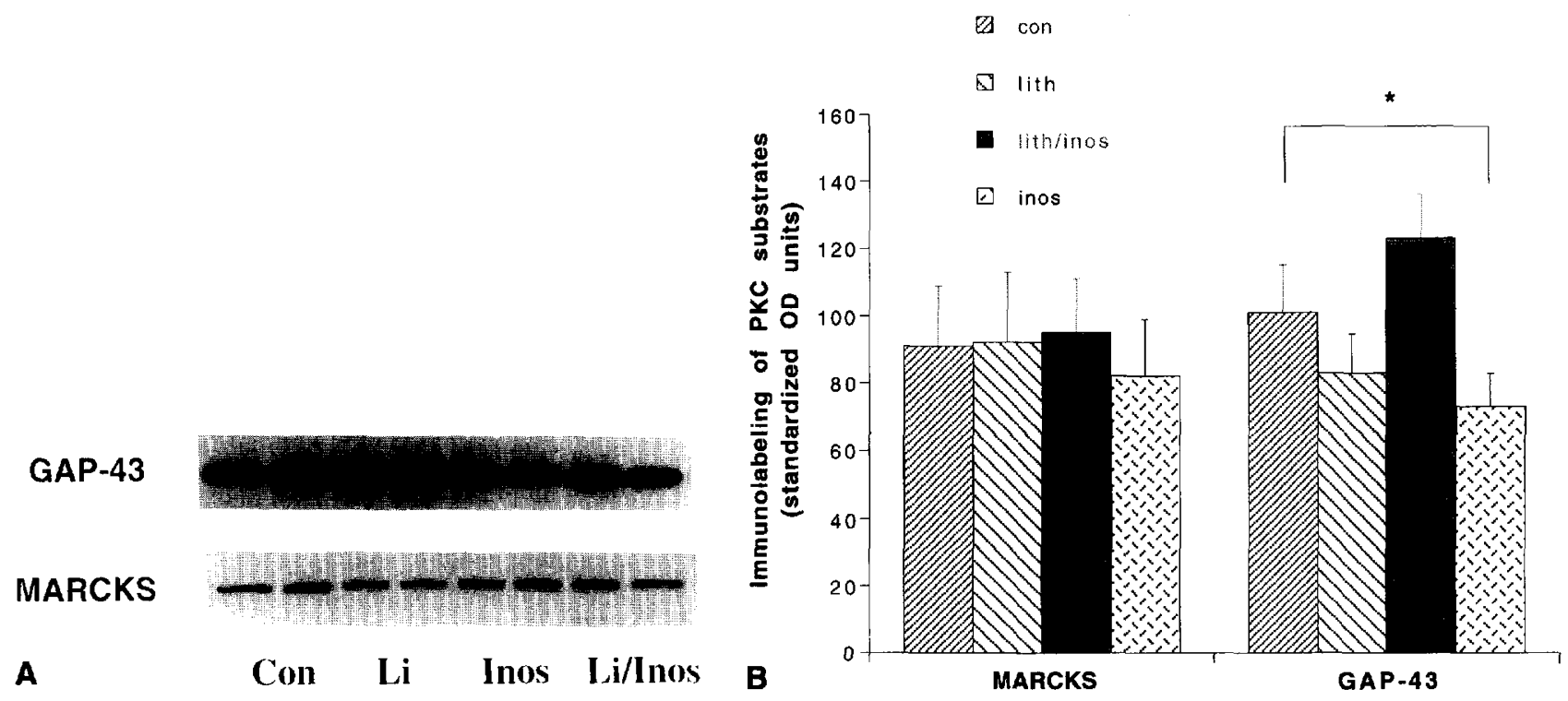

Figure 2. Effects of chronic lithium and/or myo-inositol on immunolabeling of PKC substrates in the frontal cortex. Rats were treated with lithium \pm myo-inositol for 3 weeks, and cytosolic and membrane fractions from frontal cortices were prepared and subjected to SDS-PAGE on $10 \%$ polyacrylamide gels. Proteins thus resolved were electrophoretically transferred to nitrocellulose membranes; blots were incubated overnight with anti-MARCKS or anti GAP-43 antibodies. Quantitation of the immunoblots was performed by densitometric scanning of the autoradiograms, and the immunolabeling was normalized to a pooled standard. (A) Representative immunoblot of MARCKS and GAP-43. (B) Effects of chronic lithium \pm myo-inositol administration on immunolabeling of MARCKS and GAP-43 $\left(n=8\right.$ in each group). ${ }^{*}$, Near-significant interaction between lithium and inositol on the levels of GAP-43 in the particulate fraction (two-way ANOVA; $F_{1,28}=3.86 ; p \pm .06$ ). $L i$, lithium treated; Con, control; Inos, myo-inositol treated; MARCKS, myristoylated alanine-rich C kinase substrate; GAP-43, growthassociated protein.

\section{DISCUSSION}

In the present study, we have obtained partial support for the proposal that the action of chronic lithium on
PKC isozymes and substrates may stem initially from its potent effect in inhibiting the recycling of inositol; however, the effects observed with the chronic ICV administration of myo-inositol alone make an unequivocal

\section{[32P]} ADPR

A
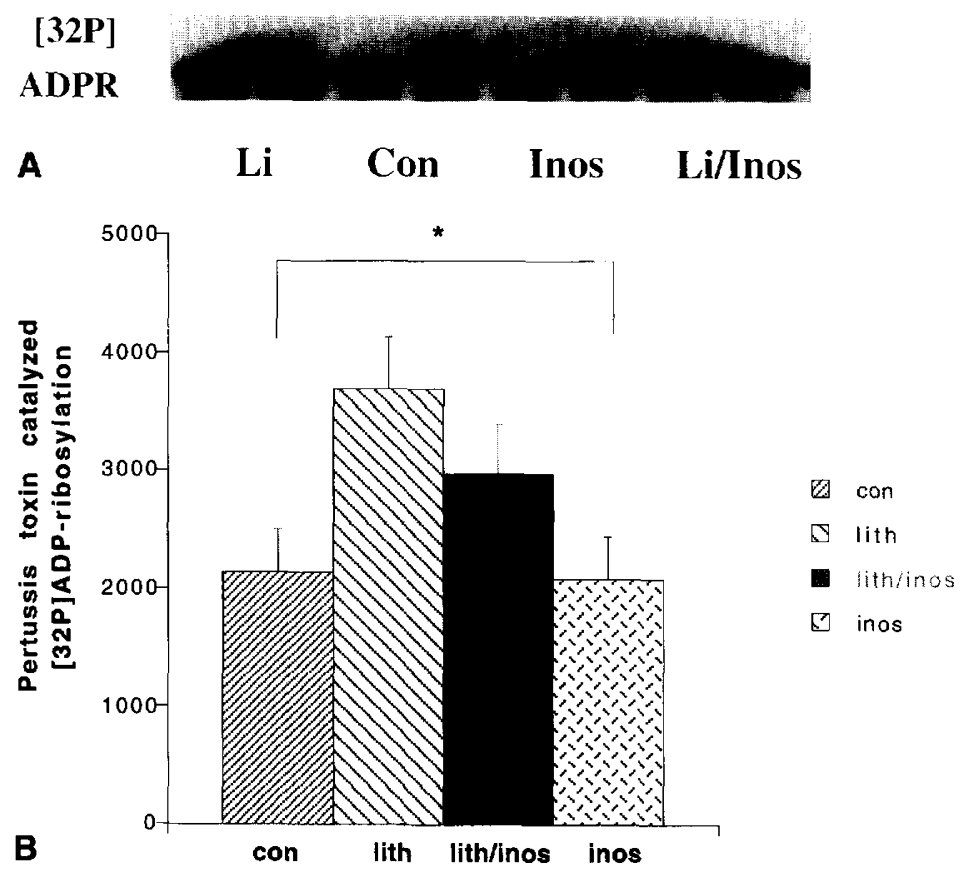

Figure 3. Effects of chronic lithium on PT-catalyzed [22 P]ADP-ribosylation in the hippocampus. Rats were treated with lithium \pm myo-inositol for 3 weeks, membranes were prepared from hippocampi and were subjected to PT-catalyzed labeling with $10 \mu \mathrm{M}\left[\alpha^{32} \mathrm{P}\right]-\mathrm{NAD}(25 \mathrm{Ci} / \mathrm{mmol})$ and activated toxin. Pertussis toxin catalyzed [ $\left.{ }^{32} \mathrm{P}\right] \mathrm{ADP}$ ribosylation of all samples was performed in duplicate. Labeled membranes were washed in ice-cold buffer, proteins were solubilized and subjected to SDS-PAGE on $10 \%$ polyacrylamide gels; the gels were dried and exposed to an X-ray film with an intensifying screen at $-80^{\circ} \mathrm{C}$. Quantitation of [ $\left.{ }^{32} \mathrm{P}\right]$ incorporation was performed by densitometric scanning of the autoradiograms. (A), Representative autoradiogram of PT-catalyzed [32P]ADP-ribosylation. (B), Effects of chronic lithium \pm myo-inositol administration on PT-catalyzed [ $\left.{ }^{32} \mathrm{P}\right] \mathrm{ADP}$-ribosylation $(n=$ 8 in each group). ${ }^{*}$ Highly significant interaction between chronic lithium and inositol treatment on PT-catalyzed [ $\left.{ }^{32} \mathrm{P}\right] \mathrm{ADPR}$ in the hippocampus (twoway ANOVA; $\left.F_{1,28}=9.40 ; P<.004\right)$. I $\left.^{32} P\right] A D P R$, PTcatalyzed [ $\left.{ }^{32} \mathrm{P}\right] \mathrm{ADP}$-ribosylation; $\mathrm{L} i$, lithium treated; Con, control; Inos, myo-inositol treated. 
Figure 4. Effects of chronic lithium on PT-catalyzed [22P]ADP-ribosylation in the frontal cortex. Rats were treated with lithium \pm myo-inositol for 3 weeks, membranes were prepared from hippocampi and were subjected to PT-catalyzed labeling with $10 \mu \mathrm{M}\left[\alpha^{-32} \mathrm{P}\right]-\mathrm{NAD}(25 \mathrm{Ci} / \mathrm{mmol})$ and activated toxin. Labeled membranes were washed in ice-cold buffer, proteins were solubilized and subjected to SDS-PAGE on $10 \%$ polyacrylamide gels; the gels were dried and exposed to X-ray film with an intensifying screen at $-80^{\circ} \mathrm{C}$. Quantitation of $\left.{ }^{32} \mathrm{P}\right]$ incorporation was performed by densitometric scanning of the autoradiograms. (A), Representative autoradiogram of PT-catalyzed [ $\left.{ }^{32} \mathrm{P}\right] \mathrm{ADP}-$ ribosylation. (B), Effects of chronic lithium \pm myoinositol administration on PT-catalyzed [2P]ADPribosylation ( $n=8$ in each group). * Significant interaction between chronic lithium and inositol treatment on PT-catalyzed [ $\left.{ }^{32} \mathrm{P}\right] \mathrm{ADPR}\left(F_{1,28}=6.74 ; p<\right.$ .05). [ $\left.{ }^{32} P\right] A D P R$, PT-catalyzed [32P]ADP-ribosylation; Li, lithium treated; Con, control; Inos, myoinositol treated.

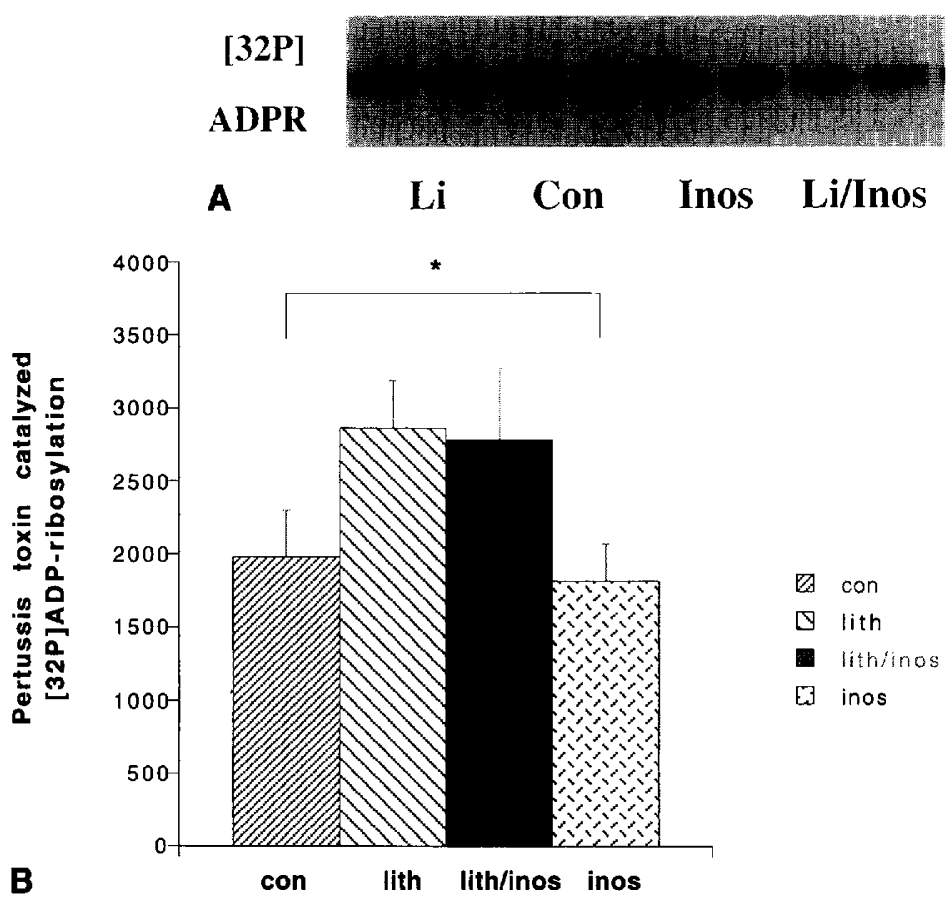

The mechanism(s) by which chronic lithium treatment produces the isozyme-specific effects on PKC and regulates the levels of its major substrates, MARCKS and GAP-43, remains to be fully elucidated. PKC subspecies exhibit subtle differences in their biochemical characteristics and cellular locations and also differ in their susceptibility to degradation by phorbol esters, which potently cause activation and membrane-insertion of the isozymes, thereby rendering them more susceptible to proteolytic degradation (Young et al. 1987; Kishimoto et al. 1989; Isakov et al. 1990; Borner et al. 1992). It is also noteworthy that exposure of neuroblastoma cells (Leli and Hauser 1992) or PC12 cells (Jope $\mathrm{RS}$, personal communication) to $1 \mathrm{mM}$ lithium in vitro produces isozyme-selective decreases in PKC $\alpha$ and (in the case of PC12 cells) PKC $\epsilon$; these resutls are strikingly similar to those we have observed in ex vivo (that is after chronic treatment of the animals) studies (Manji et al. 1993; Masana et al. 1995, under revision). Lithium, possibly via its inhibition of inositol-1-phosphatase, increases the levels of diacylglycerol (DAG), an endogenous activator of $\mathrm{PKC}$, and its metabolites (phosphatidic acid and cytidine monophosphate-phosphatidic acid) (Drummond and Raeburn 1984; Downes and Stone 1986; Godfrey et al. 1989; Brami et al. 1991; Varney et al. 1992). Because prolonged PKC activation is often followed by its proteolytic degradation (Young et al. 1987; Kishimoto et al. 1989), a prolonged increase in DAG levels by lithium may lead to an increased membrane translocation and subsequent degradation of PKC (Manji et al. 1993). Moreover, PKC activation also produces a reduction in the levels of MARCKS (Erusalimsky et al. 
1991; Linder et al. 1992), suggesting that the reduction in hippocampal MARCKS observed following chronic lithium administration may also be mediated via a lithium-induced increase in DAG levels and subsequent activation of PKC isozymes; the results of the present study support such a contention.

An intriguing aspect of the study worthy of further comment is the observation that chronic administration of myo-inositol alone also had significant effects on PKC isozymes and substrates; the myo-inositol effects were generally similar to those observed with lithium administration alone, but rather than being additive, coadministration of lithium and myo-inositol attenuated lithium's effects. Interestingly, a somewhat analogous interaction between lithium and inositol has been observed in behavioral studies (Kofman et al. 1991); thus these investigators found that, although inositol alone produced a nonsignificant decrease in rodent rearing behavior, it markedly attenuated the lithium-induced decreases in rearing. Recently, oral inositol has been reported to have antidepressant effects (Levine et al. 1995) and antipanic effects in humans. It may seem somewhat paradoxical that lithium effects are reversible with inositol, and inositol itself has psychoactive effects and biochemical effects in the same direction. However, inositol may have effects at several points in the PI cycle; thus, it can be a substrate for PI synthase and conceivably increase PI levels when PI synthase is not fully saturated. It may inhibit phospholipase $C$ or regulate it in a complex fashion and thus modulate second-messenger function. In addition, the literature on the use of inositol to enhance surfactant synthesis in neonates with respiratory distress syndrome suggests that inositol can shift the ratio of phosphatidylcholine and phosphatidylinositol in membranes. Recent studies by Eichberg and co-workers (Zhu and Eichberg 1990; Eichberg et al., 1993) also have provided evidence that a specific pool of myo-inositol used for PI synthesis is depleted in sciatic nerves of diabetic rats; interestingly, in such model systems, there is a decrease in PKC activity, and most important for the present study, PKC activity is increased in response to exogenously applied myo-inositol, possibly because of an increase in PI-derived DAG levels (Kim et al. 1991; discussed in Eichberg et al. 1993).

Although the results of the present study as well as the studies outlined suggest that chronic myo-inositol has significant effects on PKC, the precise mechanisms underlying these effects remain to be elucidated. One potential mechanism is the "driving" of the PI cycle and the generation of DAG by high concentrations of exogenously applied myo-inositol. DAG is metabolized via phosphatidic acid to the liponucleotide CDP-diacylglycerol (CDP-DAG), which in the presence of inositol is converted to PI in a reaction catalyzed by PI synthase (Agranoff et al. 1958). The latter enzyme is reported to have a relatively low affinity for inositol (Benjamins and Agranoff 1969; Ghalayani and Eichberg 1985), and thus under conditions where inositol concentrations are reduced, PI synthesis may be compromised. There is at present, however, no evidence to suggest that PI synthase is not saturated with myo-inositol under "basal" conditions (that is, conditions in which PI synthesis has not been compromised by the administration of a druglike lithium), making it difficult to reconcile the proposal that myo-inositol can drive PI turnover under such conditions. It remains possible, however, that inositol concentrations are lower in biochemically and behaviorally relevant brain compartment (e.g., in neurons) or alternatively that there is a PI-synthase enzyme that has a higher $K_{\mathrm{m}}$ for inositol (Imai and Gershengorn 1987). Indeed, it has been proposed that much of the variation seen in different experimental preparations for the study of brain lithium effects may be explained on the basis of differences in inositol content (Sherman et al. 1985; Stubbs and Agranoff 1993).

An additional hypothesis is suggested by the fact that myo-inositol is an endogenous organic osmolyte and a very hyperosmotic solution of inositol was used chronically in this study. The possibility that at least some of the effects of myo-inositol administration alone may be mediated by its osmolytic effects is suggested by a recent study that compared the effects of epi-inositol (an isomer that cannot be used for phosphoinositide synthesis) on the lithium/pilocarpine seizure paradigm (Williams and Jope 1995); however, because two other isomers of inositol, namely, chiro (Kofman et al. 1993) or scyllo (Tricklebank et al. 1991) are ineffective in this model, the effects of epi-inositol do not appear simply to represent nonspecific osmotic effects. It is thus clear that further studies are necessary to elucidate the mechanim(s) by which myo-inositol produces its various biological effects.

In the present study, similar to what we have previously observed (Masana et al. 1995, under revision), we found significantly higher levels of PT-catalyzed [ $\left.{ }^{32} \mathrm{P}\right] \mathrm{ADP}-$ ribosylation in brain membranes prepared from rats chronically treated with lithium. Because pertussis toxin selectively ADP-ribosylates the undissociated, inactive $\alpha \beta y$ heterotrimeric form of $G_{i}$, it has been postulated that lithium inactivates $G_{i}$ via a stabilization of the inactive conformation (Hsiao et al. 1992; Masana et al. 1995, under revision). Interestingly, in the present study, there was a highly significant interaction between chronic lithium and inositol treatment with respect to PT-catalyzed [ $\left.{ }^{32} \mathrm{P}\right] \mathrm{ADPR}$ in the hippocampus, with myo-inositol markedly attenuating lithium's effects. At present, the molecular mechanism(s) underlying lithium's effects on $\mathrm{G}$ proteins remains to be fully established. There is some evidence to suggest that lithium has acute in vitro effects on G proteins (Avissar et al. 1988; Greenwood and Jope 1994), but many of chronic lithium's effects persist after washing of the membranes and are there- 
fore likely attributable to an indirect posttranslational modification of the G proteins (Jope and Williams 1994; Manji et al. 1995). Because $G$ protein function is known to be regulated by phosphorylation (Houslay 1991) and considerable evidence has shown that lithium affects PKC, one potential mechanistic explanation for lithium's effects on $G$ protein subunit dissociation is phosphorylation by PKC. The results of the present study, in which coadministration of myo-inositol attenuated lithium-induced increases in PT-catalyzed [ $\left.{ }^{32} \mathrm{P}\right] \mathrm{ADP}$ labeling provides indirect support for a role of PKC. It should be noted, however, that in contrast to the alterations of PKC isozymes observed with chronic myo-inositol alone, no such alterations in PT-catalyzed [ $\left.{ }^{32} \mathrm{P}\right] \mathrm{ADP}$ labeling were observed with chronic myo-inositol administration, precluding a simple unequivocal interpretation of the results.

In conclusion, we have shown that chronic lithium treatment produces complex effects on PKC isozymes and on the conformational state of $G_{i} / G_{0}$, and that these effects are attenuated by the coadministration of myoinositol. The precise mechanism(s) underlying these effects remains to be fully elucidated; given the critical role of PKC isozymes and G proteins in modulating intracellular cross-talk between neurotransmitter systems, and thereby the integrative functions of the CNS, these effects may play a major role in lithium's mood-stabilizing effects. Thus, future studies using the bisphosphonate inhibitors of inositol monophosphatases (Atack et al. 1993, 1994) are warranted, because they may delineate the role of inositol depletion on lithium's complex long-term effects and offer the hope for the development of more potent and more rapidly acting mood-stabilizing drugs.

\section{ACKNOWLEDGMENTS}

The authors wish to thank Dr. Robert H. Lenox and Dr. Richard S. Jope for their critical review of the manuscript and for thoughtful discussions.

\section{REFERENCES}

Agranoff BW, Bradley RM, Brady RO (1958): The enzymatic synthesis of inositol phosphatide. J Biol Chem 233:10771083

Allison JH, Stewart MA (1971): Reduced brain inositol in lithium-treated rats. Nature New Biol 233:267-268

Atack JR, Cook SM, Watt AP, Ragan CI (1993): In vitro and in vivo inhibition of inositol monophosphatase by the biphosphonate L-690,330. J Neurochem 60:652-658

Atack JR, Prior AM, Fletcher SR, Quirk K, McKernan R, Ragan CI (1994): Effects of L-690,488, a prodrug of the bisphosphonate inositol monophosphatase inhibitor L-690,330, on phosphatidylinositol cycle markers. J Pharmacol Exp Ther 270: 70-76

Avissar S, Schreiber G, Danon A, Belmaker RH (1988): Lith- ium inhibits adrenergic and cholinergic increases in GTP binding in rat cortex. Nature 331:440-442

Batty IH, Downes CP (1994): The inhibition of phosphoinositide synthesis and muscarinic-receptor-mediated phospholipase $\mathrm{C}$ activity by $\mathrm{Li}+$ as secondary, selective, consequences of inositol depletion in 1321N1 cells. Biochem J 297:529-537

Belmaker RH (1981): Receptors, adenylate cyclase, depression, and lithium. Bio Psychiatry 16:333-350

Benjamins JA, Agranoff BW (1969) Distribution and properties of of CDP-diglyceride:inositol transferase from brain. J Neurochem 16:513-527

Berridge MJ, Downes CP, Hanley MR (1982): Lithium amplifies agonist-dependent phosphatidylinositol responses in brain and salivary glands. Biochem J 206:587-595.

Berridge MJ, Downes CP, Hanley MR (1989): Neural and developmental actions of lithium: A unifying hypothesis. Cell 59:411-419.

Bersudsky Y, Vinnitsky I, Grisaru N, Kofman O, Belmaker RH (1993): Dose-response and time curve of inositol prevention of Li-pilocarpine seizures. Eur Neuropsychopharmacol 3:428-429

Borner C, Guadango SN, Fabbro D, Weinstein IB (1992): Expression of four PKC isozymes in rat fibroblasts: distinct subcellular distribution and regulation by calcium and phorbol esters. J Biol Chem 267:12892-12899

Bradford MM (1976): A rapid and sensitive method for the quantitation of microgram quantities of protein utilizing the principle of protein-dye binding. Anal Biochem 72: 223-229

Brami BA, Leli U, Hauser G (1991a): Influence of lithium on second messenger accumulation in NG108-15 cells. Biochem Biophys Res Commun 174:606-612

Brami BA, Leli U, Hauser G (1991b): Origin of the diacylglycerol produced in excess of inositol phosphates by lithium in NG108-15 cells. J Neurochem 57:(Supp) S9

Busa WB, Gimlich RL (1989): Lithium-induced teratogenesis in frog embryos prevented by a polyphosphoinositide cycle intermediate or a diacylglycerol analog. Dev Biol 132:315-324.

Casebolt TL, Jope RS (1989): Long-term lithium treatment selectively reduces receptor-coupled inositol phospholipid hydrolysis in rat brain. Biol Psychiatry 25:329-340

Downes CP, Stone MA (1986): Lithium-induced reduction in intracellular inositol supply in cholinergically stimulated parotid gland. Biochem J 234:199-204

Drummond AH, Raeburn CA (1984): The interaction of lithium with thyrotropin releasing hormone-stimulated lipid metabolism in GH3 pituitary tumor cells. Biochem J 244:129-136.

Ebstein R, Belmaker R, Grunhaus L, Rimon R (1976): Lithium inhibition of adrenaline-stimulated adenylate cyclase in humans. Nature 259:411-413

Eichberg J, Abe S, Berti-Mattera LN, Day NS, Lowery JM, Zhu X, Peterson RG (1993): Inositol and phospholipid metabolism in diabetic nerve. Diab Med 10(suppl 2):16S-20S

Erusalimsky JD, Brooks SF, Herget T, Morris C, Rozengurt E (1991): Molecular cloning and characterization of the 
acidic $80-\mathrm{kDa}$ protein kinase $\mathrm{C}$ substrate from rat brain. J Biol Chem 266:7073-7080

Fisher SK, Heacock AM, Agranoff BW (1992): Inositol lipids and signal transduction in the nervous system: An update. J Neurochem 58:18-38

Ghalayani A, Eichberg J (1985): Purification of phosphatidylinositol synthetase from rat brain by CDP-diacylglycerol affinity chromatography and properties of the purified enzyme. J Neurochem 44:175-182.

Godfrey PP, McClue SJ, White AM, Wood AJ, Grahame SD (1989): Subacute and chronic in vivo lithium treatment inhibits agonist- and sodium fluoride-stimulated inositol phosphate production in rat cortex. J Neurochem 52:498-506

Goodwin FK, Jamison KR (1990): Manic-Depressive Illness. New York, Oxford University Press

Greenwood AF, and Jope RS (1994): Brain G-protein proteolysis by calpain: Enhancement by lithium. Brain Res 636:320-326

Hallcher LM, Sherman WR (1980): The effects of lithium ion and other agents on the activity of myo-inositol-1-phosphatase from bovine brain. J Biol Chem 255:10896-10901

Houslay MD (1991): "Crosstalk": A pivotal role for protein kinase $\mathrm{C}$ in modulating relationships between signal transduction pathways. Eur J Biochem 195:9-27

Hsiao JK, Manji HK, Chen GA, Bitran JA, Risby ED, Potter WZ (1992): Lithium administration modulates platelet $\mathrm{Gi}$ in humans. Life Sci 50:227-233

Imai A, Gershengorn MC (1987): Independent phosphatidylinositol synthesis in pituitary plasma membrane and endoplasmic reticulum. Nature 325:726-728

Isakov N, McMahon P, Altman A (1990): Selective post-translational down-regulation of protein kinase $C$ isozymes in leukemic $\mathrm{T}$ cells chronically treated with phorbol ester. J Biol Chem 265:2091-2097

Janowsky DS, El-Yousef MK, Davis JM, Serkerke HJ (1972): A cholinergic-adrenergic hypothesis of mania and depression. Lancet 2:632-635

Jope RS, Williams MB (1994): Lithium and brain signal transduction systems. Biochem Pharmacol 47:429-434

Kendall DA, Nahorski SR (1987): Acute chronic lithium treatments influence agonist- and depolarization-stimulated inositol phospholipid hydrolysis in rat cerebral cortex. J Pharmacol Exp Ther 241:1023-1027

Kennedy ED, Chaliss RJ, Nahorski SR (1989): Lithium reduces the accumulation of inositol polyphosphate second messengers following cholinergic stimulation of cerebral cortex slices. J Neurochem 53:1652-1655

Kennedy ED, Challiss RAJ, Ragan CI (1990): Reduced inositol polyphosphate accumulation and inositol supply induced by lithium in stimulated cerebral cortex slices. Biochem J 267:781-786

Kim J, Rushovich EH, Thomas TP, Agranoff BW, Greene DA (1991): Diminished specific activity of cytosolic Protein kinase $\mathrm{C}$ in sciatic nerve of streptozocin-induced diabetic rats and its correction by myo-inositol. Diabetes 40:1545 -1554

Kishimoto A, Mikawa K, Hashimoto K, Yasuda I, Tanaka S, Tominaga M, Kuroda T, Nishizuka Y (1989): Limited proteolysis of protein kinase $C$ subspecies by calcium- dependent neutral protease (calpain). J Biol Chem 264:4088-4092

Kofman O, Belmaker RH (1993): Biochemical, behavioral, and clinical studies of the role of inositol in lithium treatment and depression. Biol Psychiatry 34:839-852

Kofman O, Belmaker RH, Grisaru N (1991): Myo-inositol attenuates two specific behavioral effects of acute lithium in rats. Psychopharmacol Bull 27:185-190

Kofman O, Sherman WR, Katz V, Belmaker RH (1993): Restoration of brain myo-inositol levels in rats increases latency to lithium-pilocarpine seizures. Psychopharmacology 110:229-234

Laemmli UD (1970): Cleavage of structural proteins during the assembly of the head of bacteriophage T4. Nature 227:680-684

Lenox RH, Manji HK (1995): Lithium. In CB Nemeroff and AF Schatzberg (eds), American Psychiatric Press Textbook of Psychopharmacology. Washington, DC, American Psychiatric Press, pp 303-350

Lenox RH, Watson DG, Patel J, Ellis J (1992): Chronic lithium administration alters a prominent PKC substrate in rat hippocampus. Brain Res 570:333-340.

Levine J, Barak Y, Gonsalves M, Szor H, Elizur A, Kofman O, Belmaker RH (1995): A double-blind controlled study of inositol treatment of depression. Am J Psychiatry 152: 792-794

Linder D, Gschwendt M, Marks F (1992): Phorbol esterinduced down-regulation of the $80-\mathrm{kDa}$ myristoylated alanine-rich C-kinase substrate-related protein in Swiss 3T3 fibroblasts. Inhibition by staurosporine. J Biol Chem 267:24-26

Manji HK (1992): G proteins: Implications for psychiatry. Am J Psychiatry 149:746-760.

Manji HK, Lenox RH (1994): Long-term action of lithium: A role for transcriptional and posttranscriptional factors regulated by protein kinase C. Synapse 16:11-28

Manji HK, Potter WZ, Lenox RH (1995): Signal transduction pathways: Molecular targets for lithium's actions. Arch Gen Psychiatry 52(7):531-543

Manji HK, Etcheberrigaray R, Chen G, Olds JL (1993): Lithium decreases membrane-associated protein kinase $C$ in hippocampus: Selectivity for the $\alpha$ isozyme. J Neurochem 61:2303-2310

Mork A, Geisler A, Hollund P (1992): Effects of lithium on second messenger systems in the brain. Pharmacol Toxi$\operatorname{col} 71(\mathrm{~S} 1): 4-17$

Pontzer NJ, Crews FT (1990): Desensitization of muscarinic stimulated hippocampal cell firing is related to phosphoinositide hydrolysis and inhibited by lithium. J Pharmacol Exp Ther 253:921-929

Rana RS, Hokin LE (1990): Role of Phosphoinositides in Transmembrane Signaling. Physiol Rev 70:115-164

Sherman WR, Munsell LY, Gish BG, Honchar MP (1985) Effects of systemically administered lithium on phosphoinositide metabolism in rat brain, kidney and testis. J Neurochem 45:2639-2646

Stubbs EB, Argranoff BW (1993): Lithium enhances muscarinic receptor-stimulated CDP-diacylglycerol formation in inositol-depleted SK-N-SH neuroblastoma cells. J Neurochem 60:1292-1299 
Sun GY, Navidi M, Yoa FG (1992): Lithium effects on inositol phospholipids and inositoil phosphates: Evaluation of an in vivo model for assessing polyphosphoinositide turnover in brain. J Neurochem 58:290-297

Tricklebank MD, Singh L, Jackson A (1991): Evidence that a proconvulsant action of lithium is mediated by inhibition of myo-inositol phosphatase in mouse brain. Brain Res 558:145-148.

Ui M (1990): Pertussis Toxin as a valuable probe for G protein involvement in signal transduction. In. J Moss and $M$ Vaughn (eds), ADP-Ribosylation Toxins and G Proteins. Washington, DC, American Society for Microbiology

Varney MA, Godfrey PP, Drummond AH, Watson SP (1992): Chronic lithium treatment inhibits basal and agonist- stimulated responses in rat cerebral cortex and GH3 pituitary cells. Mol Pharmacol 42:671-678

Watson SP, Shipman L, Godfrey PP (1990): Lithium potentiates agonist formation of [3H]CDP-diacylglycerol in human platelets. Eur J Pharmacol 188:273-276

Williams MB, Jope RS (1995): Modulation by inositol of cholinergic- and serotonergic-induced seizures in lithium treated rats. Brain Res 685:169-178

Young S, Parker PJ, Ullrich A, Stabel S (1987): Down-regulation of Protein kinase $C$ is due to an increased rate of degradation. Biochem J 24:775-779

Zhu X, Eichberg J (1990): A myo-inositol pool utilized for phosphatidylinositol synthesis is depleted in sciatic nerve from rats with streptozotocin-induced diabetes. Proc Natl Acad Sci U S A 87(24):9818-9822 